\title{
Analysis of the WN star WR 102c, its WR nebula, and the associated cluster of massive stars in the Sickle Nebula ${ }^{\star}$
}

\author{
M. Steinke ${ }^{1,2}$, L. M. Oskinova ${ }^{1}$, W.-R. Hamann ${ }^{1}$, A. Sander ${ }^{1}$, A. Liermann ${ }^{3}$, and H. Todt ${ }^{1}$ \\ 1 Institute of Physics and Astronomy, University of Potsdam, 14476 Potsdam, Germany \\ e-mail: [msteinke; lida] @astro.physik.uni-potsdam.de \\ 2 I. Physikalisches Institut der Universität zu Köln, Zülpicher Straße 77, 50937 Köln, Germany \\ ${ }^{3}$ Leibniz Institute for Astrophysics Potsdam (AIP), An der Sternwarte 16, 14482 Potsdam, Germany
}

Received 3 November 2015 / Accepted 13 January 2016

\section{ABSTRACT}

\begin{abstract}
Context. The massive Wolf-Rayet type star WR 102c is located near the Quintuplet Cluster, one of the three massive star clusters in the Galactic centre region. Previous studies indicated that WR 102c may have a dusty circumstellar nebula and is among the main ionising sources of the Sickle Nebula associated with the Quintuplet Cluster.

Aims. The goals of our study are to derive the stellar parameters of WR 102c from the analysis of its spectrum and to investigate its stellar and nebular environment.

Methods. We obtained observations with the ESO VLT integral field spectrograph SINFONI in the $K$-band, extracted the stellar spectra, and analysed them by means of stellar atmosphere models.

Results. Our new analysis supersedes the results previously reported for WR 102c. We significantly decrease its bolometric luminosity and hydrogen content. We detect four early OB type stars close to WR 102c. These stars have radial velocities similar to that of WR 102c. We suggest that together with WR 102c these stars belong to a distinct star cluster with a total mass of $\sim 1000 M_{\odot}$. We identify a new WR nebula around WR 102c in the SINFONI map of the diffuse Br $\gamma$ emission and in the HST Pa $\alpha$ images. The Br $\gamma$ line at different locations is not significantly broadened and similar to the width of nebular emission elsewhere in the H II region around WR 102c.

Conclusions. The massive star WR 102c located in the Galactic centre region resides in a star cluster containing additional early-type stars. The stellar parameters of WR 102c are typical for hydrogen-free WN6 stars. We identify a nebula surrounding WR 102c that has a morphology similar to other nebulae around hydrogen-free WR stars, and propose that the formation of this nebula is linked to interaction of the fast stellar wind with the matter ejected at a previous evolutionary stage of WR 102c.
\end{abstract}

Key words. stars: early-type - stars: individual: WR 102c - stars: Wolf-Rayet - Galaxy: center - HII regions - infrared: stars

\section{Introduction}

The advent of IR observations led to the discovery of many massive stars in the central part of our Galaxy, mainly congregated in three massive star clusters, but also scattered in the field (e.g. Figer et al. 1999b; Homeier et al. 2003). The relatively isolated massive stars in the Galactic centre (GC) region have not been extensively observed and are as yet poorly understood (among recent results, see e.g. Dong et al. 2015; Habibi et al. 2014; Oskinova et al. 2013).

To study two such relatively isolated Wolf-Rayet (WR) stars, WR 102c and WR 102ka (known as the Peony star), we obtained mid-IR spectra using the IRS spectrograph on board the Spitzer telescope (Houck et al. 2004). These observations lead to the detection of dusty circumstellar nebulae around these objects (Barniske et al. 2008). This was an unexpected discovery, since it was previously believed that dust cannot survive in the immediate vicinity of hot stars of spectral type WN. However, since then, dusty IR nebulae have been discovered around more WNtype stars (Gvaramadze et al. 2009, 2014; Burgemeister et al. 2013). Even more importantly, such dusty nebulae have become an observational "smoking gun" in the search for new WR stars (Flagey et al. 2011; Mauerhan et al. 2011; Wachter et al. 2010).

\footnotetext{
* The scientific results reported in this article are based on observations obtained during the ESO VLT program 383.D-0323(A).
}

Barniske et al. (2008) have performed an analysis of the near-IR $K$-band spectrum of the Peony star by means of the nonLTE stellar atmosphere code PoWR (e.g. Hamann \& Koesterke 1998b; Gräfener et al. 2002). It has been shown that this object is among the most luminous, and initially most massive stars in the Galaxy. However, at the time of their paper good quality spectra of WR $102 \mathrm{c}$ were not available and only crude qualitative conclusions could be made about this source.

To remedy this situation and to study the Peony star and WR 102c in more detail, we obtained integral field spectra of these stars and their nebulae with the Spectrograph for Integral Field Spectroscopy in the Near Infrared (SINFONI). In the previous paper (Oskinova et al. 2013, herafter Paper I) we analysed the Peony star WR 102ka and its surrounding. In this paper we concentrate on the analysis of WR 102c.

WR 102c is located in the neighbourhood of the Quintuplet Cluster, in a large H II region seen in the IR images as extended diffuse emission and called the Sickle Nebula (see Fig. 1). The star was discovered during a survey by Figer et al. (1999b), who classified it as WN6 subtype. The goal of our present analysis is to obtain the parameters of WR 102c by means of spectral modelling, and to unravel its evolutionary history and possible ties with the Quintuplet Cluster. We study the environment of WR 102c by means of integral field spectroscopy to search for other massive stars that, together with WR 102c, may form a 


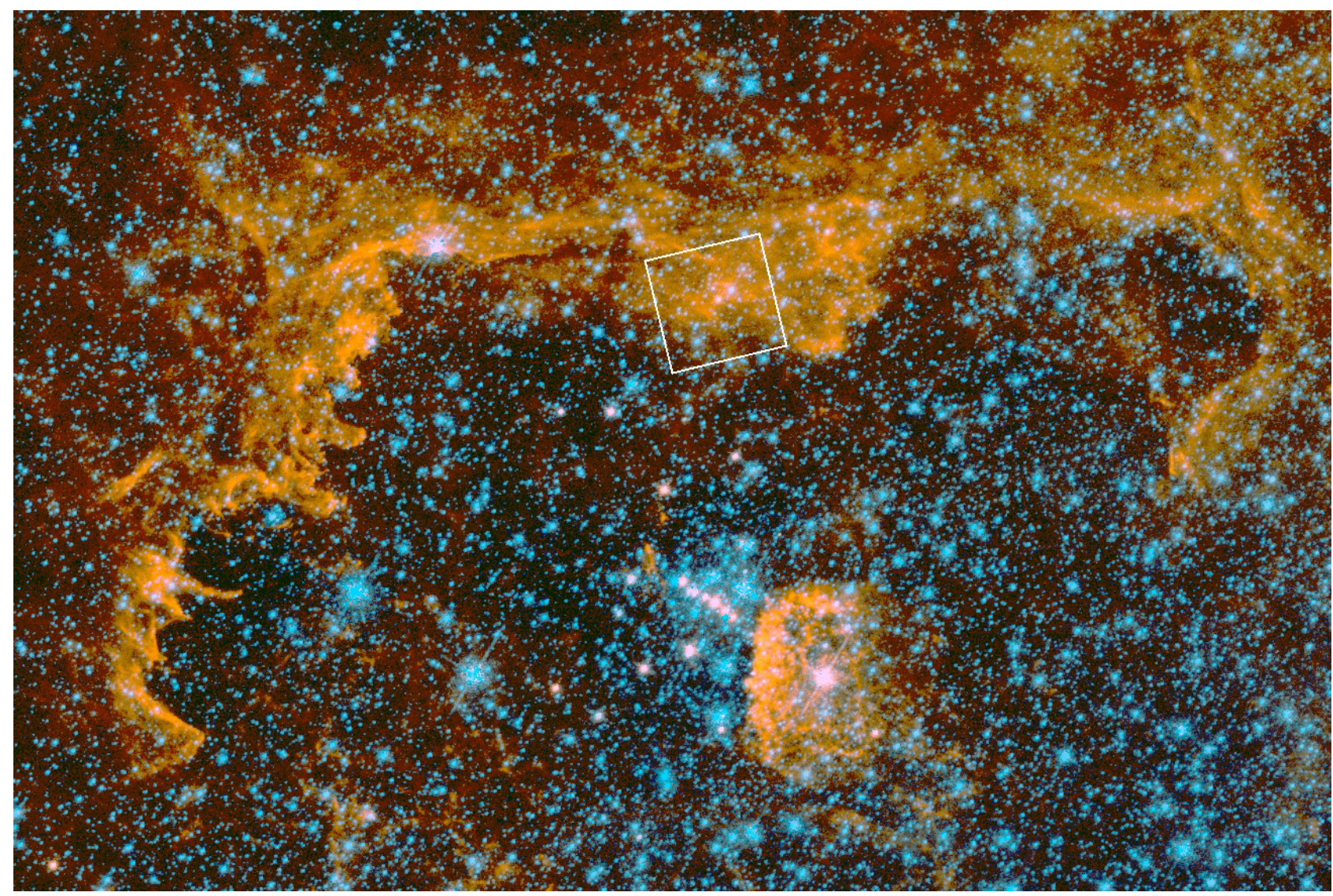

Fig. 1. HST image of the Quintuplet Cluster and the Sickle Nebula. Image size is $4.4 \times 22^{\prime} 9$. The horizontal axis is parallel to Galactic longitude. The net Paschen- $\alpha$ image is in red (see details in Dong et al. 2011), the NICMOS NIC3 F190N image in green, and the NICMOS NIC3 F187N image in blue (continuum and line filter for Pa $\alpha$ ). The white box around WR $102 \mathrm{c}$ marks the field of our SINFONI mosaic. This field is $\approx 1^{\prime}$ from the centre of the Quintuplet Cluster, which is seen as a group of bright blue stars just below the image centre close to the Pistol Nebula (identified by its characteristic shape). The image is based on the data obtained in the Paschen- $\alpha$ survey of the Galactic centre (Wang et al. 2010).

massive distinct subcluster. We also study the part of the Sickle Nebula that is directly influenced by ionising radiation from WR 102c. Throughout the paper we adopt distance modulus $D M=14.5$ mag (Reid 1993).

Special interest in WR 102c is also motivated by the recent report by Lau et al. (2016) who have identified a dusty helix shape filament in the vicinity of the Quintuplet Cluster. Lau et al. propose that this dusty filament is a precessing, collimated outflow from WR 102c. Furthermore, it has been suggested that WR102c has a gravitationally bound compact binary companion with an orbital period $>800$ days. This hypothesis potentially makes WR 102c a very rare and interesting object that deserves a thorough study.

The SINFONI observations and data reduction are presented in Sect. 2. The circumstellar nebula around WR 102c is discussed in Sect. 3. The spectral analysis of WR 102c is described in Sect. 4. The objects detected near WR 102c, in particular a subcluster of early-type stars, are discussed in Sect. 5, while the summary and the conclusions are drawn in Sect. 6.

\section{Observations and data reduction}

The data used in this work were obtained with the ESO VLT UT4 (Yepun) telescope between April 29 and May 19, 2009. The observations were performed with the integral field spectrograph SINFONI (Eisenhauer et al. 2003; Bonnet et al. 2004) yielding a three-dimensional data cube with two spatial dimensions and one spectral dimension. The $K$-band (1.95-2.45 $\mu \mathrm{m})$ grating with resolving power $R \approx 4000$ was used. The spatial scale was chosen as 0.25 per pixel. The total observation consists of a mosaic of seven pointings (observational blocks), covering $\approx 24^{\prime \prime} \times 23^{\prime \prime}(\approx 0.9 \mathrm{pc} \times 0.9 \mathrm{pc})$ centred on WR 102c (see Fig. 2$)$.

The adaptive optics facility could not be used since there is no sufficiently bright reference star in the neighbourhood. The $\log$ of the observations is given in Table 1. ABBA (science field - sky - sky - science field) cycles were performed at each pointing. Each field was observed with a total exposure time of $300 \mathrm{~s}$, usually as the sum of two detector integrations of $150 \mathrm{~s}$ each, except for the fields 361404 and 361410 where we had to split the integrations into $30 \mathrm{~s}$ intervals to avoid saturation of the brightest objects. To obtain flux calibrated spectra, standard stars were observed at similar airmass and in the same mode as the science targets. The seeing was between 0 . $^{\prime} 6$ and $11^{\prime \prime} 1$, limiting the angular resolution of our observations.

The data reduction was performed with the SINFONI pipeline version 2.32 (EsoRex version 3.9.6) using additional cosmic removal from L.A.cosmic (van Dokkum 2001) and selfwritten tools in Interactive Data Language (IDL). The procedure was the same as performed for the Peony star and we refer to Sect. 2 of Paper I for details.

The flux-calibrated three-dimensional data cubes of the individual fields were combined to a grand mosaic cube. This cube was "collapsed", i.e. summed over all wavelengths, to obtain a pass-band image for the purpose of point source detection. 
Table 1. Log of observations.

\begin{tabular}{|c|c|c|c|c|c|c|}
\hline $\begin{array}{l}\text { Block } \\
\text { (ID) }\end{array}$ & $\begin{array}{l}\text { Date } \\
2009\end{array}$ & $\begin{array}{c}\text { RA (J2000) } \\
17^{\mathrm{h}} 46^{\mathrm{m}}\end{array}$ & $\begin{array}{c}\operatorname{Dec}(\mathrm{J} 2000) \\
-28^{\circ} 49^{\prime}\end{array}$ & $\mathrm{SKY}^{a}$ & $\begin{array}{l}\text { Telluric } \\
\text { standard }\end{array}$ & $\begin{array}{r}\text { Average } \\
\text { seeing ["] }\end{array}$ \\
\hline 361398 & April 29 & $11^{\mathrm{s}} .10$ & -0.9 & B1 & HIP 83861 & 1.05 \\
\hline 361400 & April 29 & 10.56 & -0.9 & B2 & HIP 83861 & 0.98 \\
\hline 361402 & April 29 & $11^{\mathrm{s}} .47$ & $06 . .3$ & B1 & HIP 83861 & 1.01 \\
\hline 361404 & May 17 & $10^{\varsigma} .93$ & $06 . ' 9$ & B2 & HIP 88857 & 0.64 \\
\hline 361406 & May 17 & $10 \$ 37$ & $06 . " 3$ & B1 & HIP 85138 & 0.75 \\
\hline 361408 & May 19 & $11^{\mathrm{s}} .51$ & $144^{\prime \prime} 0$ & B2 & HIP 86951 & 0.57 \\
\hline 361410 & May 19 & $10^{5} .97$ & $144^{\prime \prime} 0$ & B1 & HIP 84435 & 0.65 \\
\hline
\end{tabular}

Notes. ${ }^{(a)}$ SKY fields B1 and B2 are centred at $17^{\mathrm{h}} 46^{\mathrm{m}} 18^{\mathrm{s}} .53,-28^{\circ} 48^{\prime} 5^{\prime \prime} \cdot 7$ and $17^{\mathrm{h}} 46^{\mathrm{m}} 15^{\mathrm{s}} .46,-28^{\circ} 48^{\prime} 54^{\prime \prime}$, , respectively.

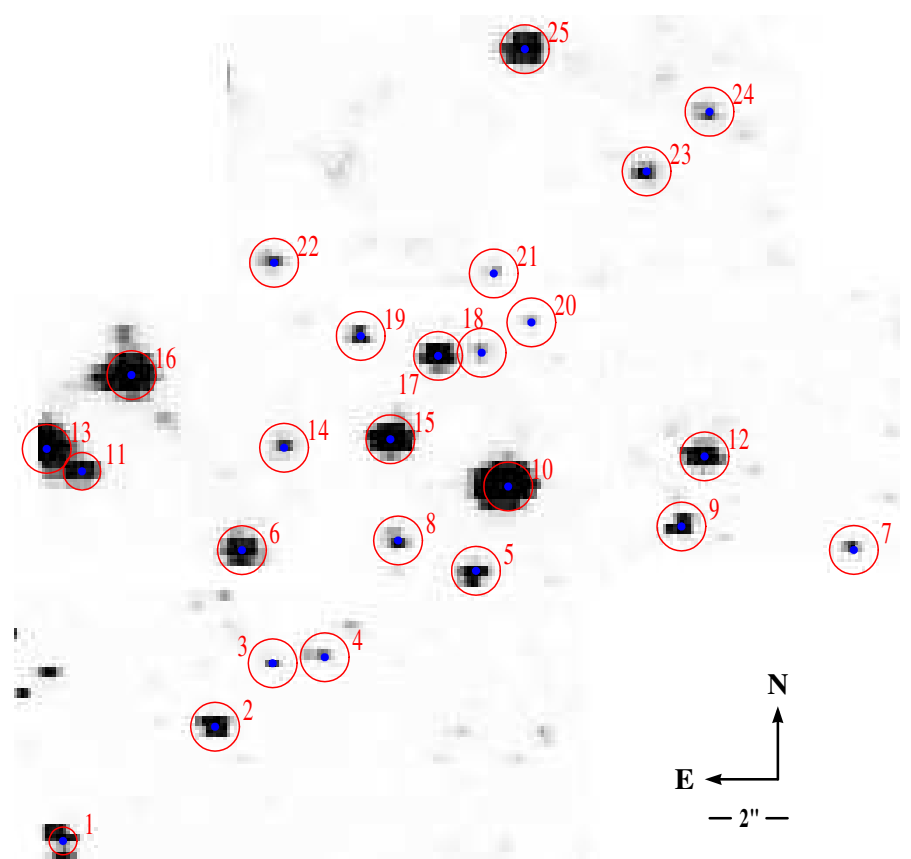

Fig. 2. Image of the collapsed grand cube (see text for details). The detected sources (open circles) are labelled with their sequential numbers (see Table 2). The image size is $23^{\prime \prime} 6 \times 23^{\prime \prime} 1(0.92 \times 0.90 \mathrm{pc})$. North is up and east is to the left.

Our observations are sensitive down to an apparent magnitude of $K_{\mathrm{s}}=14.8 \mathrm{mag}$. With the reddening towards WR $102 \mathrm{c}$ (see Sect. 4 ), the extinction in the $K$-band amounts to about 3 mag. Thus, at the distance of $8 \mathrm{kpc}$, our observations are sensitive to absolute $K$-band magnitudes $<-2.8 \mathrm{mag}$.

We securely detect 25 stellar point sources in the observed field (see Fig. 2). Their coordinates, spectral types, and radial velocities are given in Table 2 . The $K_{\mathrm{s}}$-band magnitudes were calculated from the flux-calibrated spectra, using the 2MASS filter transmission function (Skrutskie et al. 2006). The radial velocities were derived from the Doppler shift of prominent lines (except for WR 102c, which has a wind-dominated spectrum), namely the first ${ }^{12} \mathrm{CO}$ band heads for cool stars and hydrogen $\mathrm{Br} \gamma$ and helium lines for the OB-type stars. Further details are given in Sects. 4 and 5.

\section{Circumstellar nebula around WR 102c}

From the analysis of mid-IR observations of WR 102c with the Spitzer and MSX telescopes, Barniske et al. (2008) detected a dusty circumstellar nebula heated by the intense radiation of the

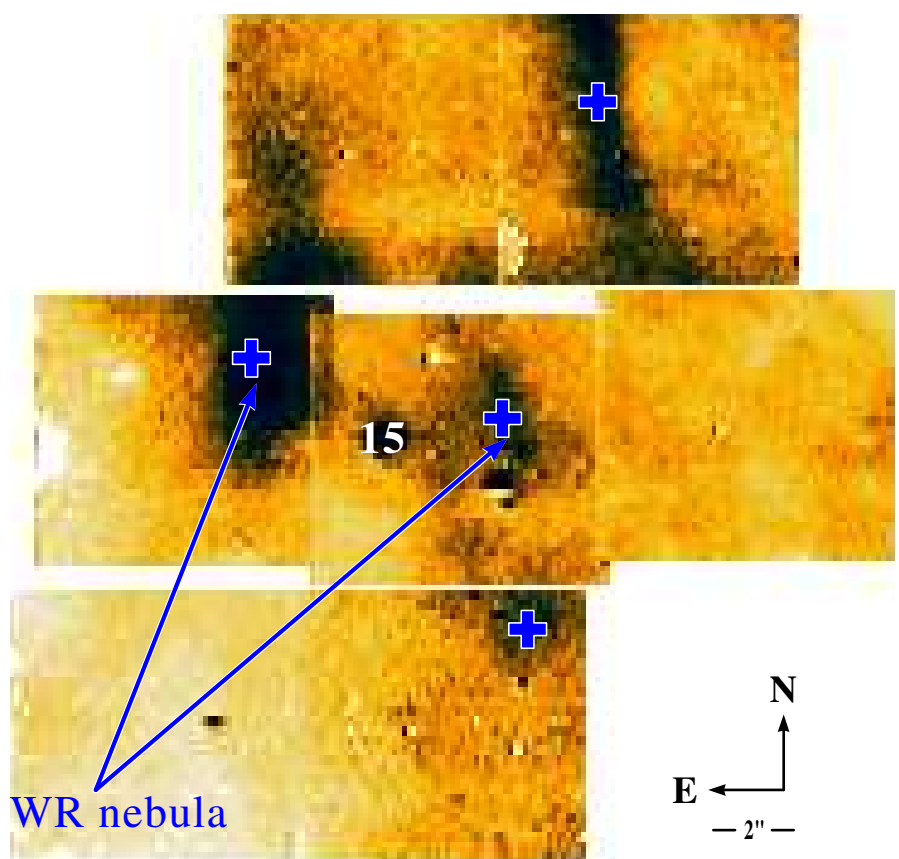

Fig. 3. Negative image at the wavelength of the $\mathrm{Br} \gamma$-line. The maximum emission (darker areas in the false-colour image) is $\approx 2 \mathrm{mJy} / \operatorname{arcsec}^{2}$ while the lowest emission (lighter areas) is $\approx 0.3 \mathrm{mJy} / \mathrm{arcsec}^{2}$. The blue arrows point out the position of the circumstellar WR 102c-nebula. Source 15 (WR 102c) is indicated in the centre of the nebula as are the positions (small crosses) of our extracted spectra (cf. Fig. 4).

WR star. However, the spatial resolution of these instruments was not sufficient to directly image this nebula. Our near-IR SINFONI data have a much better resolution. Hence, to study the nebular emission we created a narrow band image in the Bry line. A bipolar or ring structure centred on WR 102c (source 15) is clearly seen in this image (see Fig. 3). The radius of the nebula is $\approx 4^{\prime \prime}(0.15 \mathrm{pc})$. As the field is quite crowded with stars, it is difficult to conclude whether the nebula is circular or bipolar. To measure its expansion velocity, the spectra were extracted at different locations. As can be seen in Fig. 4, the nebular Br $\gamma$ lines are neither shifted nor broadened by more than $\approx 80 \mathrm{~km} \mathrm{~s}^{-1}$ (SINFONI's spectral resolution). This upper limit on the expansion velocity indicates that the nebula is not expanding quickly, opposite to what would be expected if the observed bipolar structure were the result of a fast collimated outflow.

Nebulae with various morphologies are often observed around WR stars. The mechanisms of nebula formation are linked to stellar evolution: when a massive star leaves the main sequence, it loses a large amount of matter. In the luminous blue 


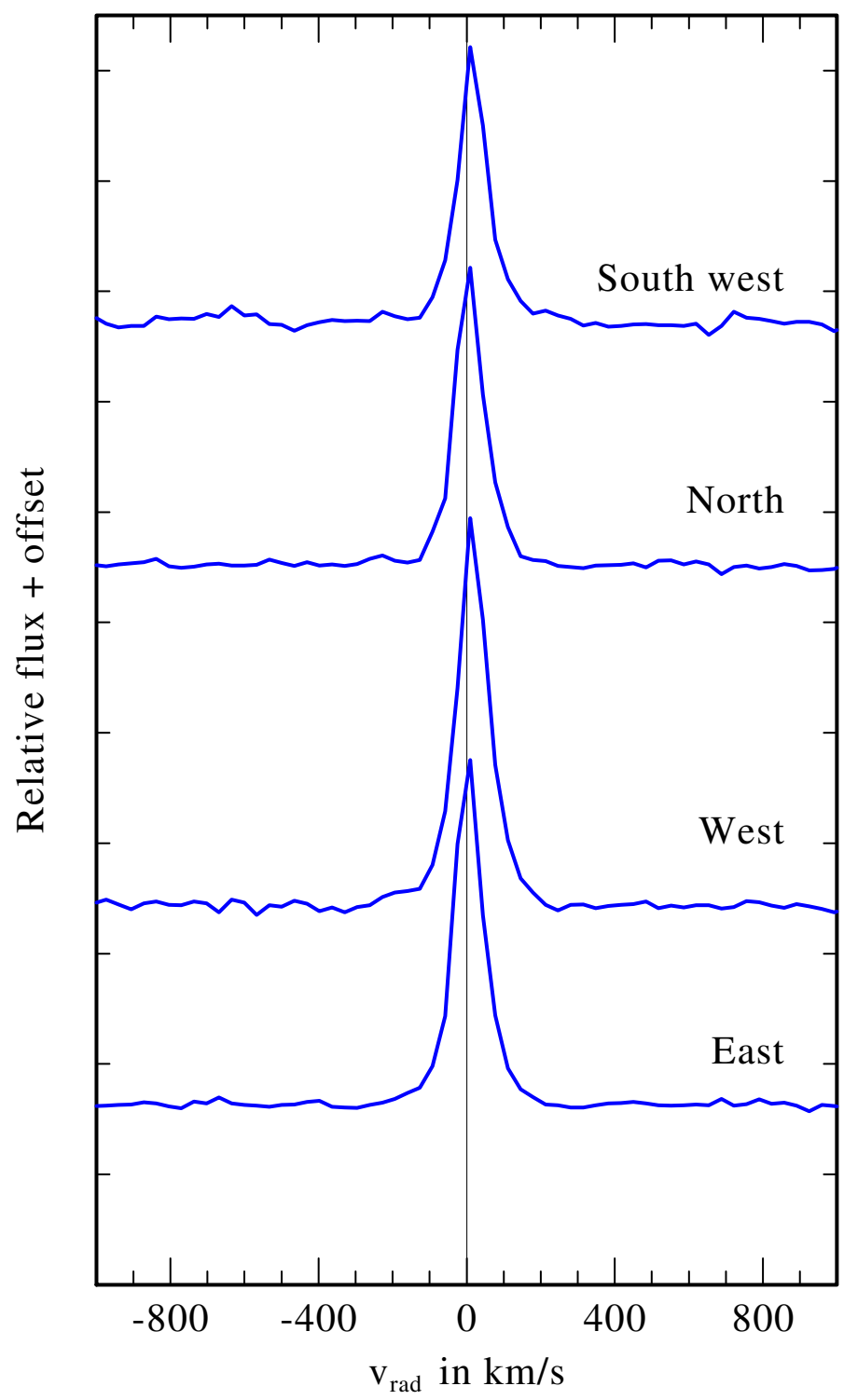

Fig. 4. Observed $\operatorname{Br} \gamma$ line in the spectrum of the WR 102c-nebula at different star-free locations. The spectra (as labeled) were taken at the following distance and direction from WR $102 \mathrm{c}$ east at $4^{\prime \prime}(0.15 \mathrm{pc})$, west at $3^{\prime \prime}(0.11 \mathrm{pc})$, north at $10^{\prime \prime}(0.38 \mathrm{pc})$ south-west at $6^{\prime \prime}(0.23 \mathrm{pc})$, cf. Fig. 3 for the positions.

variable (LBV) or a red supergiant (RSG) phase the stellar wind is dense and slow, while at a later evolutionary stages (WN) the wind is fast. The interaction between the slow and fast moving wind creates shells that are ionised and heated by the stellar radiation (e.g. Garcia-Segura \& Mac Low 1995; Freyer et al. 2006; van Marle et al. 2007; Toalá \& Arthur 2011). A recent review on WR nebulae is given in Toalá et al. (2015) and references therein.

To explain the nature of the nebula around WR 102c seen in the $\mathrm{Br} \gamma$ image we considered the following check-list. The nebula is surrounding a WR-type star. It has a small expansion velocity similar to other WR nebulae (e.g. Marston et al. 1999). The nebula is clearly seen from its hydrogen emission (in this case $\mathrm{Pa} \alpha$ and $\mathrm{Br} \gamma$ ) and in He I maps of diffuse gas, analogously to other WR nebulae. Significantly, WR nebulae have morphologies that correlate with the spectral type of their central stars. The ring or bubble-like morphologies ( $B$-type), sometimes bipolar, are found around WNE stars with fast winds, just as in the case
Table 2. Catalogue of stellar sources detected in the observed field (within $\approx 0.9 \mathrm{pc}$ ) around WR 102c.

\begin{tabular}{|c|c|c|c|c|c|}
\hline No. & $\begin{array}{c}\text { RA } \\
17^{\mathrm{h}} 46^{\mathrm{m}} \\
\end{array}$ & $\begin{array}{c}\text { Dec } \\
-28^{\circ} 49^{\prime} \\
\end{array}$ & $\begin{array}{c}K_{\mathrm{s}} \\
{[\mathrm{mag}]}\end{array}$ & $\begin{array}{l}\text { MK } \\
\text { type }\end{array}$ & $\begin{array}{r}v_{\mathrm{rad}} \\
{\left[\mathrm{km} \mathrm{s}^{-1}\right]}\end{array}$ \\
\hline 1 & $11^{\mathrm{s}} .65$ & $177^{\prime \prime} 8$ & 12.8 & M0 & -70 \\
\hline 2 & $11^{\mathrm{s}} .38$ & $14 . .9$ & 13.0 & M1 & 95 \\
\hline 3 & $11^{\mathrm{s}} .29$ & $13 . .2$ & 14.4 & $\mathrm{~K} 1$ & -90 \\
\hline 4 & $11^{\mathrm{s}} .20$ & 13". 1 & 14.4 & K4 & 85 \\
\hline 5 & $10 \varsigma .94$ & 10.8 & 13.4 & M0 & 55 \\
\hline 6 & $11^{\mathrm{s}} .34$ & $10 ! 3$ & 13.0 & M0 & 50 \\
\hline 7 & 10 s.29 & $10^{\prime} 3$ & 14.3 & K5 & 10 \\
\hline 8 & $11^{\mathrm{s}} .07$ & 10.1 & 14.0 & K4 & 205 \\
\hline 9 & $10^{\varsigma} .58$ & 09.7 & 13.4 & M0 & 60 \\
\hline 10 & $10^{s} .88$ & $08 . .7$ & 10.3 & M5-6 & -45 \\
\hline 11 & $11^{\mathrm{s}} .69$ & $08^{\prime \prime} 3$ & 13.3 & K5 & 140 \\
\hline 12 & $10 \varsigma .55$ & $07 ! .9$ & 12.4 & M1 & 100 \\
\hline 13 & $11^{\mathrm{s}} .67$ & $07 . .7$ & 12.0 & B1-2 & 100 \\
\hline 14 & $11^{\mathrm{s}} .26$ & $07 " .7$ & 14.1 & B5 & 90 \\
\hline 15 & $11^{\mathrm{s}} .08$ & $07 " .5$ & 11.6 & WN6 & 120 \\
\hline 16 & $11^{\mathrm{s}} .53$ & $05^{\prime \prime} 8$ & 12.0 & K0 & 120 \\
\hline 17 & $11^{\mathrm{s}} .00$ & 05.3 & 12.6 & B2: & 70 \\
\hline 18 & $10^{\varsigma} .93$ & $05^{\prime \prime} .2$ & 14.3 & K3 & 270 \\
\hline 19 & $11^{\mathrm{s}} .13$ & $04 . .8$ & 13.8 & K5 & 90 \\
\hline 20 & 10.84 & $04 . .5$ & 14.8 & K5 & -250 \\
\hline 21 & $10^{\varsigma} .91$ & $03 . .2$ & 14.7 & M0 & 185 \\
\hline 22 & $11^{\mathrm{s}} .28$ & 02.9 & 14.0 & M0 & 0 \\
\hline 23 & $10^{5} .64$ & $00 .{ }^{\prime} 6$ & 13.8 & K4 & -65 \\
\hline 24 & 10.54 & $-1{ }^{\prime \prime} 0$ & 14.0 & K2 & -35 \\
\hline 25 & $10^{\varsigma} .85$ & $-2 . .6$ & 12.8 & O7? & 100 \\
\hline
\end{tabular}

of WR 102c. These characteristics lead us to conclude that the nebula around WR 102c is a typical WR nebula.

However, there are some important aspects that distinguish the WR 102c nebula. It is small, only $0.15 \mathrm{pc}$, while the typical size of WR nebulae is a few parsec. The small size of this nebula can, perhaps, be attributed to the very dense and warm environment in the vicinity of the Quintuplet Cluster. Another unusual aspect is the presence of a helix-like tail likely associated with WR 102c as found by Lau et al. (2016).

The WR 102c-nebula and helix tail can also be clearly recognised in the HST Pa $\alpha$ image (Fig. 5). Moreover, it appears that another helix-like outflow is seen within the ring-nebula, as indicated in Fig. 5. However, the $\mathrm{Pa} \alpha$ images should be interpreted with caution. These were heavily processed, as explained in depth in Dong et al. (2011). The SINFONI images do not show obvious helix-like structures associated with WR 102c.

Vink et al. (2011) have found a significant correlation between fast rotating WR stars and the subset of WR stars with ejecta nebulae. They note that these objects have only recently transitioned from a previous RSG or LBV phase and thus have not yet significantly undergone spin-down which will be caused by their intensive mass loss due to the conservation of momentum. This subset of fast rotating WR stars are the candidate $\gamma$-ray burst progenitors. Gräfener et al. (2012) have identified an incidence rate of $\sim 23 \%$ for WR stars in the Galaxy that have possible ejecta nebulae and rotate faster than the average WR stars. They note that even early-type hydrogen-free WR stars, such as WR 6 (WN4) and WR 136 (WN6) can be fast rotators, perhaps evolving through binary channels. Hence, the presence of a compact bipolar nebula and a helix-like outflow from WR 102c may indicate that this object has only recently transitioned to the WR evolutionary stage, and is still a relatively fast rotator. 


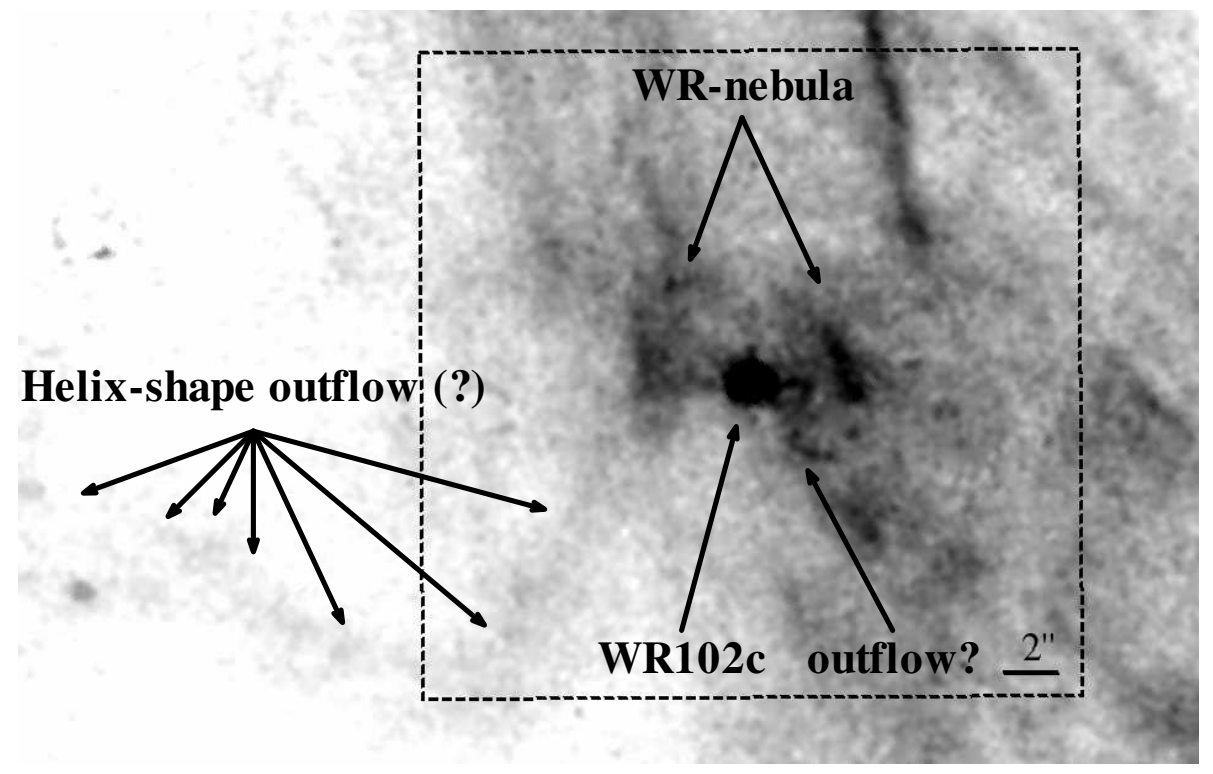

Fig. 5. HST Paschen- $\alpha$ image (Dong et al. 2011) of the ring nebula around WR 102c. The legends and the arrows indicate (i) the helixlike nebulosity detected by Lau et al. (2016); (ii) the ring WR nebula as seen in the SINFONI $\mathrm{Br} \gamma$ image in Fig. 3; (iii) a small structure that resembles a helix-like outflow. The dashed rectangle corresponds to our SINFONI mosaic field. The orientation is the same as in Figs. 2 and 3, i.e. north is up and east is to the left. The image is a zoom into the Paschen- $\alpha$ survey of the Galactic centre (Wang et al. 2010).

\section{The WN6 star WR 102c}

WR 102c was discussed in Barniske et al. (2008) who confirmed its spectral type as WN6 based on the inspection of the lowresolution spectrum shown by Figer et al. (1999b). In the present paper, we now analyse the new high-quality SINFONI spectrum of WR $102 \mathrm{c}$ in detail largely revising the estimates made in Barniske et al. (2008).

The WR 102c spectrum (Fig. 6) was analysed by means of PoWR model atmospheres ${ }^{1}$ (Hamann \& Gräfener 2004; Todt et al. 2015). The PoWR code has been used extensively to analyse the spectra of massive stars in the IR as well in the ultraviolet and optical range (e.g. Oskinova et al. 2007, 2011; Liermann et al. 2010; Sander et al. 2012; Hainich et al. 2014). The PoWR code solves the non-LTE radiative transfer in a spherically expanding atmosphere simultaneously with the statistical equilibrium equations and accounts at the same time for energy conservation. Complex model atoms with hundreds of levels and thousands of transitions are taken into account. The computations for the present paper include detailed model atoms for hydrogen, helium, carbon, oxygen, nitrogen, and silicon. Iron and iron-group elements with millions of lines are included through the concept of super-levels (Gräfener et al. 2002). The extensive inclusion of the iron group elements is important because of their blanketing effect on the atmospheric structure.

The stellar radius $R_{*}$, which is the inner boundary of our model atmosphere, corresponds by definition to a Rosseland continuum optical depth of 20 . The stellar temperature $T_{*}$ is defined by the luminosity and the stellar radius $R_{*}$ via the Stefan-Boltzmann law; i.e. $T_{*}$ denotes the effective temperature referring to the radius $R_{*}$. For the wind velocity we adopt the usual $\beta$-law with $\beta=1$ (e.g. Sander et al. 2015). Each stellar atmosphere model is defined by its effective temperature, surface gravity, luminosity, mass-loss rate, wind velocity, and chemical composition. The gravity determines the density structure of the stellar atmosphere below and close to the sonic point. If lines from the quasi-hydrostatic layers are visible in the spectrum, the analysis allows the gravity and thus the stellar mass to be derived from the pressure-broadened absorption line profiles (Sander et al. 2015).

http://www . astro.physik. uni-potsdam.de/PoWR/
For a given chemical composition and stellar temperature $T_{*}$, synthetic spectra from WR model atmospheres of different massloss rates, stellar radii, and terminal wind velocities yield almost the same emission-line equivalent widths if their "transformed radii" $R_{\mathrm{t}} \propto R_{*}\left[v_{\infty} D^{-1 / 2} \dot{M}^{-1}\right]^{2 / 3}$ (Schmutz et al. 1989) agree. To account for small-scale wind inhomogeneities, we adopt a clumping contrast of $D=4$ as a typical value for WN stars (Hamann \& Koesterke 1998a). We note that $R_{\mathrm{t}}$ is inversely correlated with the mass-loss rate, i.e. the smaller the transformed radius, the higher the density in the stellar wind.

\subsection{Temperature of WR $102 \mathrm{C}$}

Figure 6 shows the normalised spectrum of WR 102c compared to synthetic spectra. Unfortunately, it is impossible to find a model that simultaneously fits all $\mathrm{He}$ I and He II emission lines. The model for $T_{*}=75 \mathrm{kK}$ reproduces the He II emission lines perfectly, but the He I lines at $2.06 \mu \mathrm{m}$ and $2.11 \mu \mathrm{m}$ are severely underestimated (red dotted line in Fig. 6). The alternative model drawn as a green dashed line for a temperature of $T_{*}=66 \mathrm{kK}$ reproduces the $\mathrm{He} \mathrm{I}$ line at $2.06 \mu \mathrm{m}$ perfectly, but underestimates some of the He II lines. The line at $2.11 \mu \mathrm{m}$ is actually not only composed of He I, but is also blended with N III. The corresponding transitions are not fully included in our atomic data, which might explain the mismatch.

Hence, the stellar temperature remains uncertain in the range $T_{*}=66 \mathrm{kK}$ to $75 \mathrm{kK}$. Moreover, if the star is a fast rotator it could lead to variations of the wind properties between poles and equator.

It is not possible to establish the rotation rates of WR stars from spectroscopic analysis of the near-infrared only. For example, our test calculations show that even the unrealistically high photospheric rotation velocities up to $v_{\text {rot }} \sin i=1000 \mathrm{~km} \mathrm{~s}^{-1}>$ $v_{\text {crit }}$ would not leave any noticeable imprint on the $K$-band spectrum as long as the wind does not corotate (see discussion in Shenar et al. 2014). We do not observe signs of such extreme wind corotation in WR 102c.

Fortunately, the impact of the $T_{*}$ uncertainty on the implied mass-loss rate and luminosity is relatively small (see Table 3 ). In particular, the effect on $T_{2 / 3}$, i.e. the effective temperature referring to the radius of Rosseland optical depth $\tau_{\text {Ross }}=2 / 3$, is 


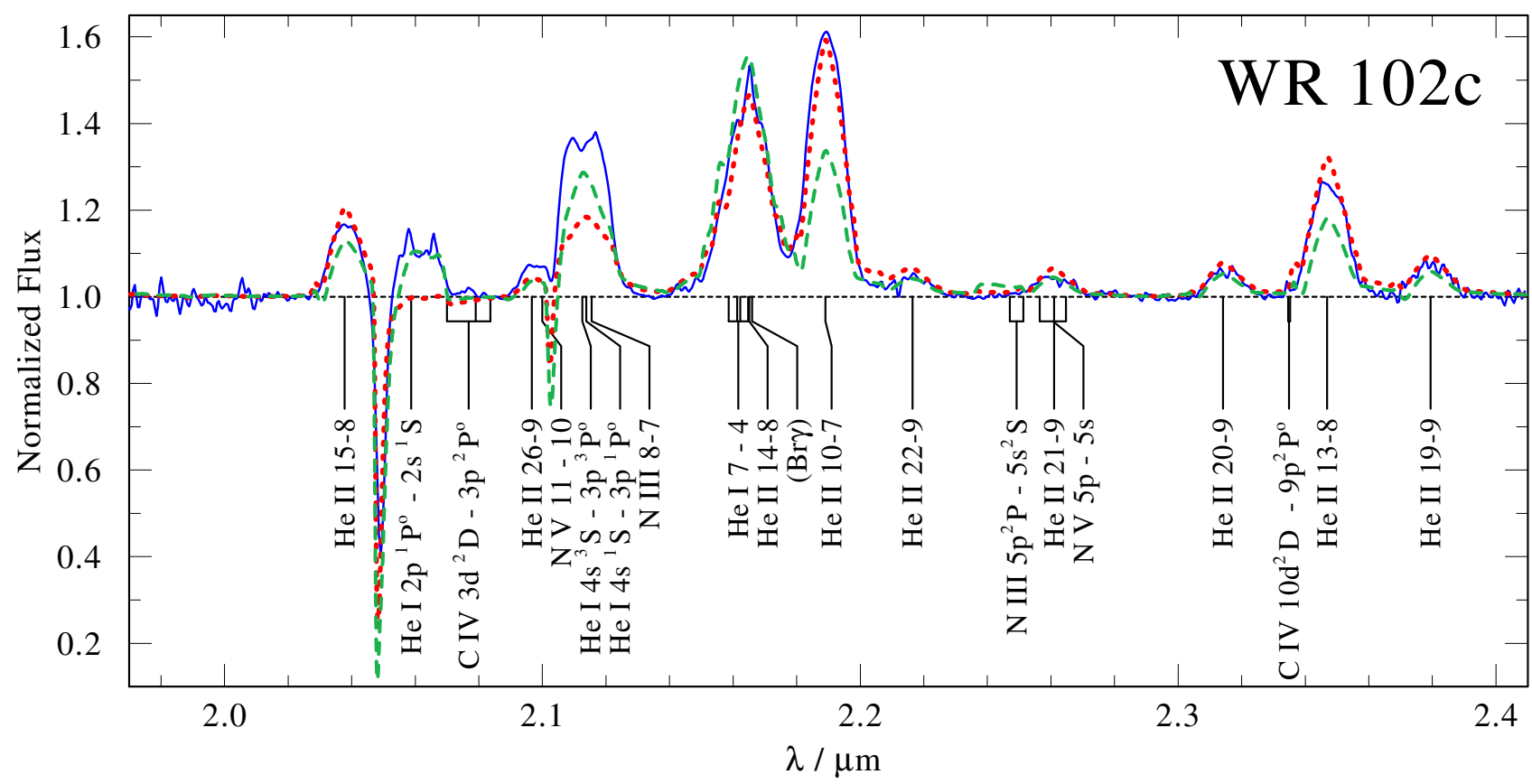

Fig. 6. Normalised $K$-band spectrum of WR 102c (solid blue), compared to two PoWR models: $T_{*}=75.0 \mathrm{kK}, \log L / L_{\odot}=5.57, \log \dot{M}=-4.17$ (red dotted line); $T_{*}=66.0 \mathrm{kK}, \log L / L_{\odot}=5.51, \log \dot{M}=-4.08$ (green dashed line). The prominent spectral features are identified.

Table 3. Stellar parameters of WR 102c.

\begin{tabular}{lr}
\hline \hline Spectral type & WN6 \\
$\log L / L_{\odot}$ & $5.5 \ldots 5.7$ \\
$T_{*}[\mathrm{kK}]$ & $66 \ldots 75$ \\
$T_{2 / 3}[\mathrm{kK}]$ & $33 \ldots 37$ \\
$\log M\left[M_{\odot} \mathrm{yr}^{-1}\right]$ & $-4.17 \ldots-4.09$ \\
$\log \Phi_{\mathrm{Ly}}\left[\mathrm{s}^{-1}\right]$ & $49.0 \ldots 49.3$ \\
$v_{\infty}\left[\mathrm{km} \mathrm{s}^{-1}\right]$ & 1600 \\
$M_{\text {ini }} / M_{\odot}$ & $\sim 40$ \\
$v_{\text {rad }}\left[\mathrm{km} \mathrm{s}^{-1}\right]$ & $120 \pm 40$ \\
$A_{K}[\mathrm{mag}]$ & 2.8 \\
\hline
\end{tabular}

hardly affected. This invariance reflects the parameter degeneracy of very dense stellar winds as discussed in e.g. Hamann et al. (2006).

Another speculative explanation for the inconsistent spectral fit could be the presence of a cooler, unresolved companion. A useful empirical diagnostic of WR-binaries is provided by X-ray observations. While single WR stars are relatively faint X-ray sources (Ignace et al. 2000; Oskinova et al. 2003), the WRtype binaries usually display colliding wind phenomena and are bright, detectable X-ray sources even in the GC region (Law \& Yusef-Zadeh 2004; Oskinova 2005). WR 102c was not detected in deep X-ray surveys of the Quintuplet Cluster (Wang et al. 2006). This non-detection provides evidence against the binarity hypothesis.

\subsection{Abundances}

Barniske et al. (2008) constrained the hydrogen abundance in WR $102 \mathrm{c}$ as being below $20 \%$ by mass. The new data allow this limit to be adjusted to $<5 \%$. If the hydrogen content were higher, it would contribute significantly to the emission at $\lambda=2.16 \mu \mathrm{m}$ $(\mathrm{He} I \mathrm{II}+\mathrm{Br} \gamma)$. Thus, our new analysis confirms that WR 102c is a virtually hydrogen-free WNE star.
In the observed spectrum, all clearly detectable metal lines (of nitrogen and carbon) are blended with helium lines (e.g. $\mathrm{N} \mathrm{V}$ at $\lambda 2.1 \mu \mathrm{m}$ or C IV at $\lambda 2.08 \mu \mathrm{m}$ ). For our models we adopted a mass fraction of $1.5 \%$ for nitrogen, which is typical for Galactic WN stars. The carbon abundance is constrained to $\ll 1 \%$, otherwise carbon emission lines would be visible (e.g. C IV at $\lambda=2.07-2.08 \mu \mathrm{m})$. Hence, we set the carbon abundance to a mass fraction of $10^{-4}$.

\subsection{Bolometric luminosity of WR $102 \mathrm{c}$}

In their discovery paper, Figer et al. (1999b) assigned a $K_{\mathrm{s}}$-band magnitude of $11.6 \mathrm{mag}$ to WR $102 \mathrm{c}$. This value was revised by Barniske et al. (2008) who erroneously used the $K_{\mathrm{s}}$-band magnitude of a nearby bright star (source 10 in Fig. 2) from the 2MASS and the Spitzer IRAC point source catalogues and adopted $K_{\mathrm{s}}=9.3 \mathrm{mag}$ for WR $102 \mathrm{c}$. This resulted in an overestimated stellar bolometric luminosity. The new SINFONI data allow us to unambiguously identify WR 102c in the near-IR image (source 15 in Fig. 2) with $K_{\mathrm{s}}=11.6 \mathrm{mag}$, in agreement with the original report.

To put strong constraints on the stellar luminosity, we compared the model stellar spectral energy distribution (SED) with the available photometric and spectrophotometric data. The result is shown in Fig. 7. We used the available photometry of WR 102c from Spitzer IRAC (Churchwell et al. 2009), UKIDSS (Lucas et al. 2008), and HST NICMOS (Dong et al. 2011). The SED fitting then allows the luminosity and the interstellar extinction to be adjusted by adopting the distance modulus of $D M=14.5 \mathrm{mag}$. The observed photometry of WR 102c is nicely reproduced by a model with a bolometric luminosity of $\log L / L_{\odot} \approx 5.6$ and the extinction $E_{B-V}=8.0 \mathrm{mag}$, corresponding to $A_{K} \approx 2.8 \mathrm{mag}$. The alternative, slightly cooler model $\left(T_{*}=66 \mathrm{kK}\right)$ leads to $\log L / L_{\odot} \approx 5.5$ and the same extinction. This $A_{K}$ is consistent with WR $102 \mathrm{c}$ being located in the GC region (e.g. Schultheis et al. 1999; Figer et al. 1999a). 


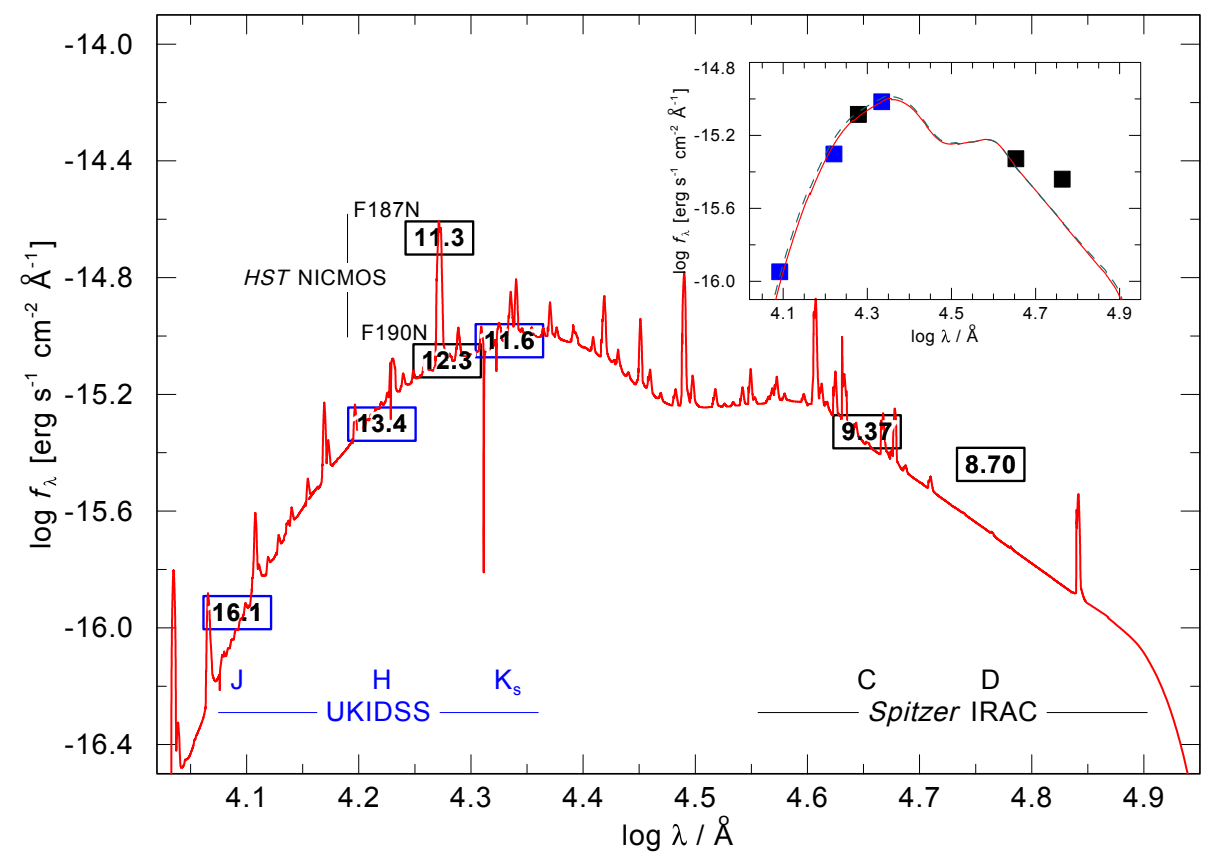

Fig. 7. Spectral energy distribution for WR 102c. The reddened spectrum of the PoWR model with $T_{*}=75 \mathrm{kK}$ is shown as a red solid line (see Table 3 for the detailed parameters) and compared to the available photometry, which is indicated by blue (UKIDSS) and black (HST NICMOS Pa $\alpha$ filters and Spitzer IRAC Channels 2 and 3) boxes. The inset in the upper right corner shows the continuum photometry marks compared to the continuum SED for the two alternative models used in Fig. 6 with $T_{*}=66 \mathrm{kK}$ (green dashed curve) and $75 \mathrm{kK}$ (red solid curve), respectively. The overall SED fit is hardly affected by the temperature uncertainty. We note that in the mid-IR the stellar SED is below the photometric observation, attributed to the emission from a warm dusty nebula (Barniske et al. 2008).
WR 102c is highly reddened and, if present, anomalous extinction may lead to an uncertainty in the estimates of stellar luminosity. To investigate this problem we did a thorough check of the various reddening laws using $R_{V}$ and $E_{B-V}$ provided by the extinction functions of Moneti et al. (2001), Cardelli et al. (1989), and Fitzpatrick \& Massa (2009). We found that the spectral energy distribution is best reproduced by an extinction of $E(B-V)=8.0 \pm 0.3$ mag using the Moneti et al. (2001) and Cardelli et al. (1989) functions, while $E(B-V)=8.7 \pm 0.4 \mathrm{mag}$ fits best for the Fitzpatrick \& Massa (2009) function, resulting in the uncertainty in the luminosity $\log L / L_{\odot}=5.5 \ldots 5.7$.

As a next step, we varied the $R_{V}$ from the commonly used value $R_{V}=3.1$ by +0.5 and +1.0 for each of the three extinction laws. The curves were then scaled in luminosity to match the $K$-band photometry. However, in all cases the reddening using the standard value $R_{V}=3.1$ provides the best description of the stellar SED and matches the photometry marks better. Thus, we conclude that there are no reasons to suspect an anomalous reddening for WR $102 \mathrm{c}$.

Our models predict that WR $102 \mathrm{c}$ produces $\log \left(\Phi_{\mathrm{Ly}}\right)=$ $49.0 \ldots 49.3\left[\mathrm{~s}^{-1}\right]$ hydrogen ionising photons. This compares well with the value of 49.45 derived from radio observations of the Sickle Nebula by Lang et al. (1997). Thus, although our revised number of ionising photons is lower than the value of Barniske et al. (2008), WR 102c can still be considered the main ionising source of the Sickle nebula. However, the Pa $\alpha$-image of the GC region (Fig. 1) does not show a fully ionised hole around WR 102c, in contrast to the region around the Quintuplet Cluster. This could mean that our WR star is located in the foreground of the Sickle nebula.

\subsection{Evolutionary status of WR $102 \mathrm{c}$}

The derived stellar luminosity and temperature allow us to evaluate the evolutionary status of WR 102c. In Fig. 8 the position of WR 102c in the HR diagram is compared with evolutionary tracks from Ekström et al. (2012) for rotating stars of solar metallicity. WR $102 \mathrm{c}$ is nicely located at the position of WNE stars on a track with an initial mass of $40 M_{\odot}$. In the case of a rotating model, the age of WR $102 \mathrm{c}$ is $\$ 6 \mathrm{Myr}$, while assuming evolution without rotation would yield $\lesssim 4$ Myr. Both estimates assume single star evolution. With its estimated age of 4-6 Myr, WR 102c would have completed at least one orbit around the GC (Stolte et al. 2014).

Assuming single star evolution and taking into account the ratios between numbers of stars with different spectral types, Liermann et al. $(2012,2014)$ constrained the age of the Quintuplet Cluster to $3 \pm 0.5 \mathrm{Myr}$. It was noticed that all WN stars in the Quintuplet have late spectral types (WN9h) and all WC stars have WC8-9 spectral types (Liermann et al. 2009). The evolutionary channels that lead to the production of late WC stars are not yet understood (see extensive discussion on this subject in Sander et al. 2012). The WC stars in the Quintuplet are severely enshrouded by dust (Moneti et al. 2001), and some of them are confirmed binaries (Tuthill et al. 2006). The importance of binary channels in the evolution of massive stars is well recognised (e.g. Vanbeveren et al. 2007). Schneider et al. (2014) used their binary evolution code to model the observed present-day mass functions of the Quintuplet Cluster and estimated its age as $4.8 \pm 1.1 \mathrm{Myr}$ if binary channels are included. Hence, within the uncertainties the age of WR 102c is comparable to the age of the Quintuplet Cluster

WR $102 \mathrm{c}$ is located $\approx 2.5 \mathrm{pc}\left(\approx 1^{\prime}\right)$ from the core of the Quintuplet Cluster. It is quite common for a massive star in the GC to reside outside of the three known large clusters (e.g. Cotera et al. 1999; Mauerhan et al. 2010; Dong et al. 2011). There are different scenarios that can explain the origin of these isolated massive stars, for example they might have been born in relative isolation, or ejected or tidally stripped from one of the three known clusters, or they might belong to clusters that have not been discovered yet.

It has recently been suggested by Lau et al. (2016) that the evolution of WR 102c may have been affected by binary interactions, and the star could have been ejected from the Quintuplet Cluster. However, based on our analysis the measured radial velocity of WR 102c is not outstanding and compares well with the average radial velocity of the Quintuplet stars $\left(\approx 110 \mathrm{~km} \mathrm{~s}^{-1}\right.$, Liermann et al. 2012). Proper motion observations are required to establish the runaway status of WR 102c. On the other hand, Habibi et al. (2014) used their $N$-body simulations and showed 


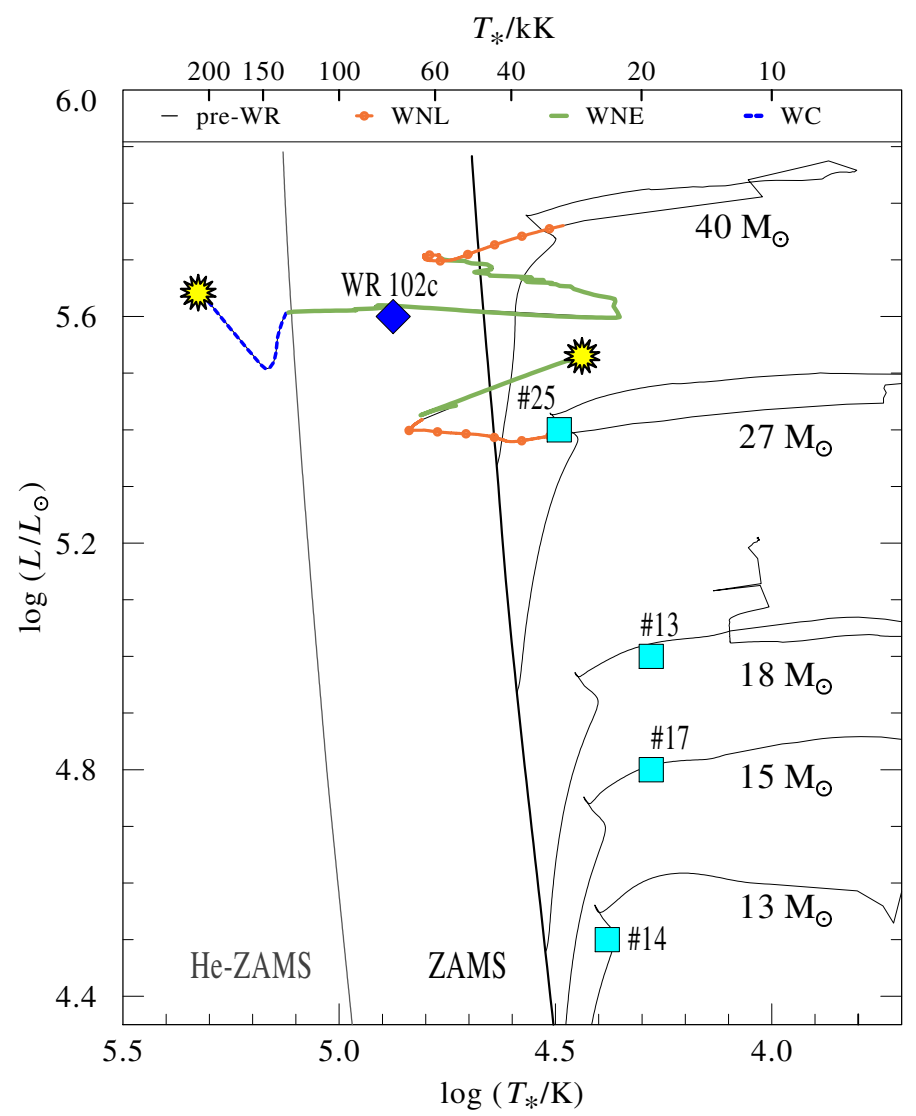

Fig. 8. HR diagram with evolutionary tracks of Ekström et al. (2012) for rotating stars of solar metallicity. The different colours identify the different stages of the stellar evolution: thin black: pre-WR phase; thick yellow: WNL phase (i.e. with hydrogen between 40-5\% mass fraction); thick green: WNE phase (hydrogen less than 5\% mass fraction); thick blue: WC phase (i.e. carbon more than $20 \%$ mass fraction). The positions of WR 102c (blue diamond) and the OB-type stars (light blue squares) are indicated as well.

that up to $80 \%$ of the isolated observed WR stars in the GC region could be explained by tidal striping. In particular, they estimate the age of the Quintuplet Cluster to 5 Myr and show that the projected tidal arms of this cluster may extend out to $60 \mathrm{pc}$ in the direction of the Sagittarius B2 region. Given the age of WR 102c and its proximity to the Quintuplet Cluster, tidal stripping is able to explain the location of WR 102c.

There is, however, another possible evolutionary path for WR 102c. Our SINFONI data revealed four OB-type stars within a projected distance of $1 \mathrm{pc}$ around WR 102c (see Sect.5). Hence, it is possible that WR $102 \mathrm{c}$ was formed together with a its own cluster, independently from the Quintuplet Cluster (see Sect. 5.3).

\section{Stars in the vicinity of WR 102c}

\subsection{Early-type stars}

Among the 25 stellar sources detected in our SINFONI observations, we found four early-type stars (see Table 2). All of these objects have counterparts in the UKIDSS Galactic Plane Survey (Lucas et al. 2008); the cross-identifications are provided in Table 4.

The $K$-band spectra of these early-type stars are shown in Fig. 9. The radial velocities measured for each star are consistent with WR 102c within the uncertainties. The extinction to each

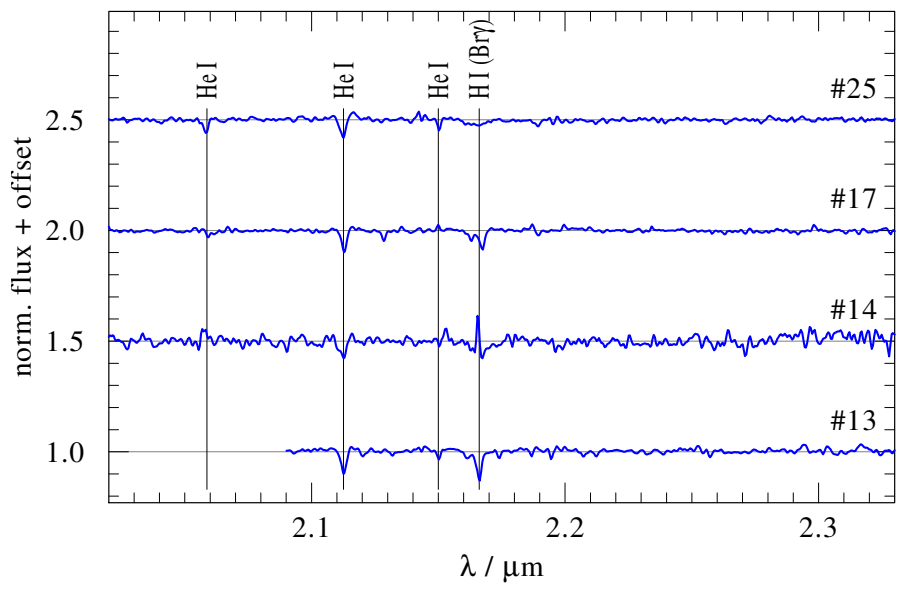

Fig. 9. SINFONI spectra of early-type stars in the WR 102c field. The number above each spectrum corresponds to the source identification in Fig. 2 and Table 2.

Table 4. Cross-correlations in WR 102c cluster with the UKIDSS catalogue. Source numbers refer to Table 2.

\begin{tabular}{cc}
\hline \hline Source No. & ID from UKIDSS catalogue \\
\hline$\# 13$ & J174611.81-284906.5 \\
$\# 14$ & J174611.34-284906.9 \\
$\# 17$ & J174611.03-284903.9 \\
$\# 25$ & J174610.87-284856.6 \\
\hline
\end{tabular}

Table 5. Stellar parameters of the OB stars in the sample.

\begin{tabular}{lrrrr}
\hline \hline Source No. & $\# 13$ & $\# 14$ & $\# 17$ & $\# 25$ \\
\hline Spectral type & B1-3 V: & B?III: & B2:III? & O9: I: \\
$\log L / L_{\odot}$ & 5.0 & 4.5 & 4.8 & 5.4 \\
$T_{*}[\mathrm{kK}]$ & 20 & 24 & $20:$ & $31:$ \\
$M_{\text {ini }}\left[M_{\odot}\right]$ & $18:$ & $13:$ & 15 & $27:$ \\
$\operatorname{lad}_{\text {rad }}\left[\mathrm{km} \mathrm{s}^{-1}\right]$ & 100 & 90 & 70 & 100 \\
$A_{K}[\mathrm{mag}]$ & 2.8 & 2.9 & 2.9 & 2.9 \\
\hline
\end{tabular}

Notes. The source number corresponds to Table 2. A colon following a value flags it as uncertain. The radial velocities have an uncertainty of $\pm 40 \mathrm{~km} \mathrm{~s}^{-1}$ due to the limited spectral resolution.

early-type star was estimated from SED fitting, and was found to be similar to the extinction towards WR 102c. Thus, we conclude that the early-type stars in the WR 102c field are likely spatially related and might even be gravitationally bound.

For the spectral classification, we use the near-IR spectral atlas of OB stars (Hanson et al. 1996, 2005). Source 25 is either a late $\mathrm{O}$ - or early B-type (super)giant. The other three objects are B-type stars. They hardly show any features in the $K$-band except for hydrogen $\mathrm{Br} \gamma$ and one or two He I lines (at $\lambda \lambda 2.06,2.11 \mu \mathrm{m}$ ). Moreover, $\mathrm{Br} \gamma$ is contaminated by nebula emission. To overcome this contamination, we subtracted the background emission from the stellar spectra. Therefore, it is difficult to determine their spectral subtypes. Nevertheless, from the wings of $\mathrm{Br} \gamma$ their luminosity classes could be constrained.

We have determined the stellar parameters of the OB-type stars in the WR 102c cluster with tentative fits of the SED and the line spectra to PoWR models. The results are compiled in Table 5. Their initial masses were estimated from the comparison with evolutionary tracks in the the HR diagram (see Fig. 8). 
Table 6. The late-type stars in the field.

\begin{tabular}{lccccrr}
\hline \hline No. & $\begin{array}{c}A_{K_{\mathrm{s}}} \\
{[\mathrm{mag}]}\end{array}$ & $\begin{array}{c}E_{B-V} \\
{[\mathrm{mag}]}\end{array}$ & $\begin{array}{c}d \\
{[\mathrm{kpc}]}\end{array}$ & $\begin{array}{c}\log L \\
{\left[L_{\odot}\right]}\end{array}$ & $\begin{array}{r}\text { MK } \\
\text { type }\end{array}$ & $\begin{array}{r}\text { lum. } \\
\text { class }\end{array}$ \\
\hline$\# 1$ & 2.9 & 8.4 & 8.0 & 2.8 & M0 & II \\
$\# 2$ & 2.2 & 6.3 & 5.8 & 2.1 & M1 & III \\
$\# 3$ & 2.1 & 6.0 & 5.5 & 1.7 & K1 & III \\
$\# 4$ & 2.1 & 6.0 & 5.5 & 1.5 & K4 & III \\
$\# 5$ & 3.3 & 9.5 & 8.1 & 2.7 & M0 & II \\
$\# 6$ & 2.9 & 8.3 & 8.0 & 2.7 & M0 & II \\
$\# 7$ & 2.9 & 8.3 & 8.0 & 2.2 & K5 & III \\
$\# 8$ & 2.8 & 8.0 & 8.0 & 2.3 & K4 & II \\
$\# 9$ & 1.9 & 5.6 & 5.3 & 1.8 & M0 & III \\
$\# 10$ & 2.9 & 8.3 & 8.0 & 3.6 & M5-6 & I \\
$\# 11$ & 2.9 & 8.2 & 8.0 & 2.6 & K5 & II \\
$\# 12$ & 2.8 & 8.0 & 8.0 & 3.0 & M1 & II \\
$\# 16$ & 2.6 & 7.5 & 7.3 & 3.1 & K0 & II \\
$\# 18$ & 2.8 & 8.0 & 8.0 & 2.3 & K3 & III \\
$\# 19$ & 2.2 & 6.3 & 5.8 & 1.9 & K5 & III \\
$\# 20$ & 2.0 & 5.7 & 6.2 & 1.5 & K5 & III \\
$\# 21$ & 2.5 & 7.3 & 7.2 & 1.8 & M0 & III \\
$\# 22$ & 2.5 & 7.3 & 7.2 & 2.1 & M0 & III \\
$\# 23$ & 2.2 & 6.3 & 5.8 & 1.9 & K4 & III \\
$\# 24$ & 2.2 & 6.3 & 5.8 & 1.9 & K2 & III \\
\hline
\end{tabular}

\subsection{Late-type stars}

In addition to the early-type stars, many point sources with latetype spectra are present in the field. The temperatures were estimated from the equivalent width of the ${ }^{12} \mathrm{CO}(2-0)$ band at $2.3 \mu \mathrm{m}$ following González-Fernández et al. (2008). On the same basis we determined the spectral types. The luminosity classes were ranked on the basis of the CO index (Blum et al. 1996, 2003). The extinction was obtained from comparison of MARCS models (Gustafsson et al. 2008) with the measured photometric values in the infrared (Lucas et al. 2008; Ramírez et al. 2008; Churchwell et al. 2009; Dong et al. 2011). The distance modulus was then inferred from this extinction by comparison with the curves published by Schultheis et al. (2014), which then also implies the luminosity. The parameters of late-type stars are given in Table 6.

The brightest star in the field (no. 10 in Table 2 and Fig. 2) is an M5-6I red supergiant. It is similar to the other late-type stars; we obtained its spectrum and fitted the SED as shown in Fig. 10. The extinction and luminosity suggest that the star most likely resides in the GC region. On the other hand, its radial velocity $\left(-45 \mathrm{~km} \mathrm{~s}^{-1}\right)$ is at odds with the radial velocities of early-type stars in this region $\left(\sim 100 \mathrm{~km} \mathrm{~s}^{-1}\right)$. The position of star 10 in the HRD would place it on a track for an initial mass of $9 M_{\odot}$. Such a star would require more than $10 \mathrm{Myr}$ to evolve to the RSG phase.

Thus, we believe that, like other late-type stars in our SINFONI field, star 10 does not belong to the WR 102c cluster, but instead represents a previous episode of star formation in the GC region. This is similar to the conclusion reached by Liermann et al. (2012) from their analysis of the Quintuplet Cluster.

\subsection{WR 102c star cluster}

The presence of early-type stars within $0.9 \mathrm{pc}$ of WR $102 \mathrm{c}$ may indicate that these objects represent an independent cluster. Alternatively, the WR 102c cluster may be related to the same starburst that produced the Quintuplet Cluster. Yet another possibility is that all the early-type stars close to WR 102c are part

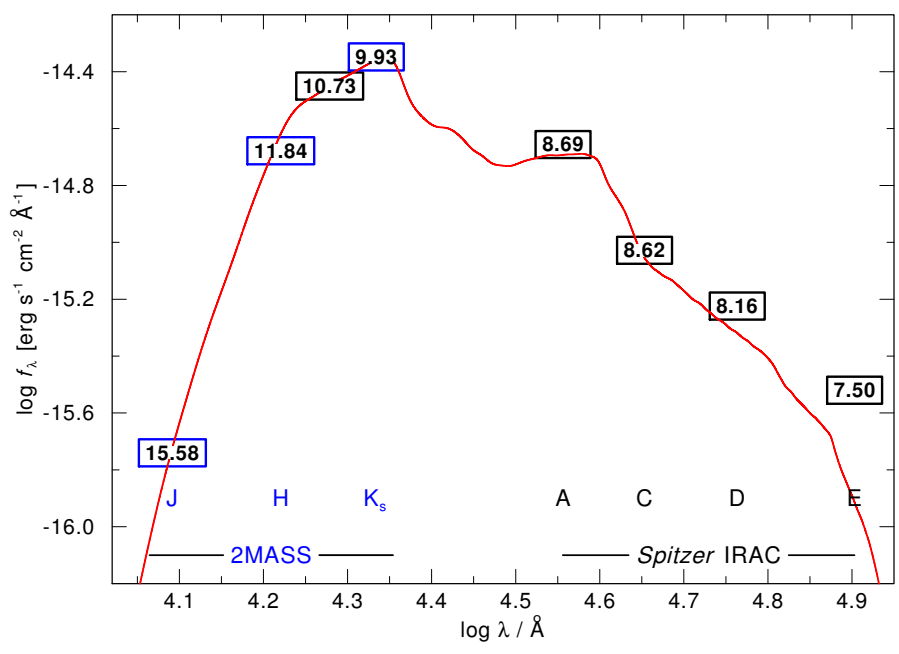

Fig. 10. Spectral energy distribution (SED) of star 10 (MSX6C G000.1668-00.0434) fitted by a MARCS model (red curve) with $T_{*}=$ $3.3 \mathrm{kK}, \log L / L_{\odot}=3.65$, and $\log g=1.0$. The boxes represent photometry from 2MASS $J, H$, and $K$ (blue, labels = magnitudes), HST NICMOS F190N (black), and Spitzer IRAC (black) in the mid-IR. The model is reddened with an extinction of $E_{B-V}=8.3 \mathrm{mag}$.

of the Quintuplet's tidal tail. Such tails have been predicted by Habibi et al. (2014) for the Arches and Quintuplet with extensions up to $1.6 \mathrm{pc}$ from the cluster centre. In any case, the presence of a cluster around WR 102c seems to be inconsistent with the scenario that WR 102c was born in the Quintuplet and ejected from there.

We used the publicly available cluster evolution code McLUSTER (Küpper et al. 2011) to estimate the mass of the WR 102c cluster. Assuming a standard Kroupa IMF (Kroupa 2001), the mass of the WR $102 \mathrm{c}$ cluster is $\sim 1000 M_{\odot}$. The simulation accurately reproduces the mass of the most massive star and the number of early-type stars we observed. Given the sensitivity of our observations ( $\left.K_{\mathrm{S}}=14-15 \mathrm{mag}\right)$ we were able to detect only stars earlier than B1-2, i.e. with masses above $10 M_{\odot}$.

Adopting an enclosed mass of $\sim 1000 M_{\odot}$ and a characteristic cluster radius $R_{0} \approx 1 \mathrm{pc}$ we estimate the velocity dispersion within the cluster stars to be only a few $\mathrm{km} \mathrm{s}^{-1}$ (following Eq. (1) in Kroupa 2002). In summary, the number of early-type stars, their ages, masses, and radial velocities consistently agree with these objects being the brightest members of a yet unknown star cluster.

\section{Summary and conclusions}

Using VLT SINFONI we obtained integral field observations of 0.25 square arcmin area around WR 102c located at $\approx 2.5 \mathrm{pc}$ (projected distance) from the Quintuplet Cluster in the $\mathrm{GC}$ region.

We identified five early-type stars (including WR 102c) in this field. Based on their age, radial velocity, and mass distribution we suggest that these are the most massive members of a bound WR 102c cluster with $M_{\mathrm{cl}} \sim 1000 M_{\odot}$. The existence of such a cluster questions the ejection scenario for the present day location of WR 102c.

The detailed analysis of the stellar spectrum and photometry of WR 102c by means of non-LTE stellar atmosphere PoWR models yielded stellar and wind parameters typical for a WN6 star (Table 3). The uncertainty of the deduced stellar parameters of WR 102c is mild and might reflect some departures of stellar 
wind from spherical symmetry, e.g. due to the fast rotation or binarity.

We produced a map of diffuse $\mathrm{Br} \gamma$ emission in the region around WR 102c. Our data clearly show a bipolar or ringshaped nebula centred on WR 102c. The same nebula is clearly seen in the images from the HST Paschen- $\alpha$ survey of the GC. According to the spectra of the nebula, the projected expansion velocity is small. We suggest that the WR 102c nebula is a typical WR nebula formed recently as the result of the interaction of winds at different evolutionary stages.

Acknowledgements. This work has extensively used the NASA/IPAC Infrared Science Archive, the NASA Astrophysics Data System, and the SIMBAD database, operated at CDS, Strasbourg, France. This publication makes use of data products from the Two Micron All Sky Survey, which is a joint project of the University of Massachusetts and the Infrared Processing and Analysis Center/California Institute of Technology, funded by the National Aeronautics and Space Administration and the National Science Foundation. Some of the data presented in this paper were retrieved from the Mikulski Archive for Space Telescopes (MAST). STScI is operated by the Association of Universities for Research in Astronomy, Inc., under NASA contract NAS5-26555. Support for MAST for non-HST data is provided by the NASA Office of Space Science via grant NNX09AF08G and by other grants and contracts. We are grateful to Dr. R. Lau for sharing with us the preprint of his paper. We thank the referee for the useful comments and suggestions. Funding for this research has been provided by DLR grant 50 OR 1302 (LMO) and DFG grant HA 1455/26 (AS).

\section{References}

Barniske, A., Oskinova, L. M., \& Hamann, W. 2008, A\&A, 486, 971 Blum, R. D., Sellgren, K., \& Depoy, D. L. 1996, AJ, 112, 1988 Blum, R. D., Ramírez, S. V., Sellgren, K., \& Olsen, K. 2003, ApJ, 597, 323 Bonnet, H., Abuter, R., Baker, A., et al. 2004, The Messenger, 117, 17 Burgemeister, S., Gvaramadze, V. V., Stringfellow, G. S., et al. 2013, MNRAS, 429,3305

Cardelli, J. A., Clayton, G. C., \& Mathis, J. S. 1989, ApJ, 345, 245

Churchwell, E., Babler, B. L., Meade, M. R., et al. 2009, PASP, 121, 213

Cotera, A. S., Simpson, J. P., Erickson, E. F., et al. 1999, ApJ, 510, 747

Dong, H., Wang, Q. D., Cotera, A., et al. 2011, MNRAS, 417, 114

Dong, H., Mauerhan, J., Morris, M. R., Wang, Q. D., \& Cotera, A. 2015, MNRAS, 446, 842

Eisenhauer, F., Abuter, R., Bickert, K., et al. 2003, in SPIE Conf. Ser. 4841, eds. M. Iye, \& A. F. M. Moorwood, 1548

Ekström, S., Georgy, C., Eggenberger, P., et al. 2012, A\&A, 537, A146

Figer, D. F., Kim, S. S., Morris, M., et al. 1999a, ApJ, 525, 750

Figer, D. F., McLean, I. S., \& Morris, M. 1999b, ApJ, 514, 202

Fitzpatrick, E. L., \& Massa, D. 2009, ApJ, 699, 1209

Flagey, N., Noriega-Crespo, A., Billot, N., \& Carey, S. J. 2011, ApJ, 741, 4

Freyer, T., Hensler, G., \& Yorke, H. W. 2006, ApJ, 638, 262

Garcia-Segura, G., \& Mac Low, M.-M. 1995, ApJ, 455, 160

González-Fernández, C., Cabrera-Lavers, A., Hammersley, P. L., \& Garzón, F. 2008, A\&A, 479, 131

Gräfener, G., Koesterke, L., \& Hamann, W.-R. 2002, A\&A, 387, 244

Gräfener, G., Vink, J. S., Harries, T. J., \& Langer, N. 2012, A\&A, 547, A83

Gustafsson, B., Edvardsson, B., Eriksson, K., et al. 2008, A\&A, 486, 951

Gvaramadze, V. V., Fabrika, S., Hamann, W.-R., et al. 2009, MNRAS, 400, 524

Gvaramadze, V. V., Chené, A.-N., Kniazev, A. Y., et al. 2014, MNRAS, 442, 929
Habibi, M., Stolte, A., \& Harfst, S. 2014, A\&A, 566, A6 Hainich, R., Rühling, U., Todt, H., et al. 2014, A\&A, 565, A27

Hamann, W.-R., \& Gräfener, G. 2004, A\&A, 427, 697

Hamann, W.-R., \& Koesterke, L. 1998a, A\&A, 335, 1003

Hamann, W.-R., \& Koesterke, L. 1998b, A\&A, 333, 251

Hamann, W.-R., Gräfener, G., \& Liermann, A. 2006, A\&A, 457, 1015

Hanson, M. M., Conti, P. S., \& Rieke, M. J. 1996, ApJS, 107, 281

Hanson, M. M., Kudritzki, R.-P., Kenworthy, M. A., Puls, J., \& Tokunaga, A. T. 2005, ApJS, 161, 154

Homeier, N. L., Blum, R. D., Pasquali, A., Conti, P. S., \& Damineli, A. 2003, A\&A, 408, 153

Houck, J. R., Roellig, T. L., van Cleve, J., et al. 2004, ApJS, 154, 18

Ignace, R., Oskinova, L. M., \& Foullon, C. 2000, MNRAS, 318, 214

Kroupa, P. 2001, MNRAS, 322, 231

Kroupa, P. 2002, MNRAS, 330, 707

Küpper, A. H. W., Maschberger, T., Kroupa, P., \& Baumgardt, H. 2011 , MNRAS, 417, 2300

Lang, C. C., Goss, W. M., \& Wood, O. S. 1997, ApJ, 474, 275

Lau, R. M., Hankins, M. J., Herter, T. L., et al. 2016, ApJ, 818, 117

Law, C., \& Yusef-Zadeh, F. 2004, ApJ, 611, 858

Liermann, A., Hamann, W., \& Oskinova, L. M. 2009, A\&A, 494, 1137

Liermann, A., Hamann, W.-R., Oskinova, L. M., Todt, H., \& Butler, K. 2010, A\&A, 524, A82

Liermann, A., Hamann, W.-R., \& Oskinova, L. M. 2012, A\&A, 540, A14

Liermann, A., Hamann, W.-R., \& Oskinova, L. M. 2014, A\&A, 563, C2

Lucas, P. W., Hoare, M. G., Longmore, A., et al. 2008, MNRAS, 391, 136

Marston, A. P., Welzmiller, J., Bransford, M. A., Black, J. H., \& Bergman, P. 1999, ApJ, 518, 769

Mauerhan, J. C., Cotera, A., Dong, H., et al. 2010, ApJ, 725, 188

Mauerhan, J. C., Van Dyk, S. D., \& Morris, P. W. 2011, AJ, 142, 40

Moneti, A., Stolovy, S., Blommaert, J. A. D. L., Figer, D. F., \& Najarro, F. 2001, A\&A, 366, 106

Oskinova, L. M. 2005, MNRAS, 361, 679

Oskinova, L. M., Ignace, R., Hamann, W.-R., Pollock, A. M. T., \& Brown, J. C. 2003, A\&A, 402, 755

Oskinova, L. M., Hamann, W.-R., \& Feldmeier, A. 2007, A\&A, 476, 1331

Oskinova, L. M., Todt, H., Ignace, R., et al. 2011, MNRAS, 416, 1456

Oskinova, L. M., Steinke, M., Hamann, W.-R., et al. 2013, MNRAS, 436, 3357

Ramírez, S. V., Arendt, R. G., Sellgren, K., et al. 2008, ApJS, 175, 147

Reid, M. J. 1993, ARA\&A, 31, 345

Sander, A., Hamann, W.-R., \& Todt, H. 2012, A\&A, 540, A144

Sander, A., Shenar, T., Hainich, R., et al. 2015, A\&A, 577, A13

Schmutz, W., Hamann, W.-R., \& Wessolowski, U. 1989, A\&A, 210, 236

Schneider, F. R. N., Izzard, R. G., de Mink, S. E., et al. 2014, ApJ, 780, 117

Schultheis, M., Ganesh, S., Simon, G., et al. 1999, A\&A, 349, L69

Schultheis, M., Chen, B. Q., Jiang, B. W., et al. 2014, A\&A, 566, A120

Shenar, T., Hamann, W.-R., \& Todt, H. 2014, A\&A, 562, A118

Skrutskie, M. F., Cutri, R. M., Stiening, R., et al. 2006, AJ, 131, 1163

Stolte, A., Hußmann, B., Morris, M. R., et al. 2014, ApJ, 789, 115

Toalá, J. A., \& Arthur, S. J. 2011, ApJ, 737, 100

Toalá, J. A., Guerrero, M. A., Ramos-Larios, G., \& Guzmán, V. 2015, A\&A, 578, A66

Todt, H., Sander, A., Hainich, R., et al. 2015, A\&A, 579, A75

Tuthill, P., Monnier, J., Tanner, A., et al. 2006, Science, 313, 935 van Dokkum, P. G. 2001, PASP, 113, 1420

van Marle, A. J., Langer, N., \& García-Segura, G. 2007, A\&A, 469, 941

Vanbeveren, D., Van Bever, J., \& Belkus, H. 2007, ApJ, 662, L107

Vink, J. S., Gräfener, G., \& Harries, T. J. 2011, A\&A, 536, L10

Wachter, S., Mauerhan, J. C., Van Dyk, S. D., et al. 2010, AJ, 139, 2330

Wang, Q. D., Dong, H., \& Lang, C. 2006, MNRAS, 371, 38

Wang, Q. D., Dong, H., Cotera, A., et al. 2010, MNRAS, 402, 895 